\title{
Place of percutaneous nephrolithotripsy (PCNL) in the management of renal calculi
}

\author{
Ketan Agarwal $^{1}$, Madhu S Agrawal ${ }^{2}$ \\ 'University of Liverpool, Liverpool, United Kingdom \\ ${ }^{2}$ S. N. Medical College, Agra, India
}

Key words: renal and upper ureteral stones; percutaneous; minimally invasive.

\section{Introduction}

Percutaneous nephrostomy is a procedure known since 1955.1 However, it was not until 1976 when the first percutaneous nephrostomy for the specific purpose of removing a kidney stone was performed by Fernstrom and Johannson [2]. Over the next few years, Smith and colleagues in the USA, Alken and Marberger in West Germany, and Wickham and colleagues in the United Kingdom, began to remove selected stones in the renal pelvis and ureter through percutaneous approach [3-5]. By the early 1980s, it was apparent that it was possible to remove renal stones safely and reliably percutaneously, not only through a previously made PCN tract, but also as a single stage procedure with considerably shortened period of hospitalization [6].

PCNL, as the procedure is commonly referred to, has now attained the status of being the 'gold standard' for removal of kidney stones of all kinds and sizes. It is now the most commonly performed procedure for renal calculi, having steadily gained in popularity at the expense of extra-corporeal shock wave lithotripsy (ESWL), another minimally invasive approach for urinary tract calculi, introduced around the same time in the early 1980 's. Open surgery for renal calculi is now more or less relegated to history in most of the modern urology centers the world over.

\section{Technique of percutaneous stone removal \\ The procedure may be divided into two parts:}

Correspondence :

Agrawal MS

Email : dr.madhu.agra@gmail.com

\section{(A) Access}

The excretory urogram (IVU) or CT urogram illustrates the accurate location of the stone within the collecting system, hence determining optimal access tract. The retroperitoneal location of the kidney permits access through a postero-lateral window through the retroperitoneum. Access should be performed under fluoroscopic or ultrasonic control. Optimum access is generally through a lower posterior calyx in most instances.

The procedure is mostly done under epidural, spinal or general anesthesia, but can also be done under intravenous sedation and local anesthesia. A $20 \mathrm{~cm}$ long 18-gauge puncture needle is placed through the flank into the pelvi-calyceal system of the kidney at the point where access is desired. A hydrophilic guide wire of .035 or .038 size is passed into the collecting system through the needle. It is very desirable that this is placed as far down the ureter as possible, or coiled in the upper calyx, in order to minimize the possibility of inadvertent loss of the tract.

The tract is enlarged by passing serial or telescopic Teflon or metal dilators co-axially over the guide wire. Dilatation proceeds under fluoroscopic control to 24 to $30 \mathrm{Fr}$ and an Amplatz sheath is passed over the last dilator, to provide direct access to the collecting system. The nephroscope (size 21-26 F) is passed through the sheath to visualize the inside of the collecting system.

\section{(B) Stone removal}

Small stones up to $8-10 \mathrm{~mm}$ size can be removed intact with forceps or basket; larger stones are fragmented by ultrasonic or pneumatic lithotripsy into removable fragments. Regardless of the energy source, the probe fragments the stone into progressively smaller pieces, which are removed as they are broken up. Stone 
removal continues until the patient is free of stone or until it becomes necessary to stop the procedure. If complete clearance is not achieved in the first sitting, the nephroscope can safely be reinserted through the same tract after 48-72 hours. At this point, the tract is matured and bleeding has almost always stopped, permitting straightforward removal of the remaining calculi and fragments.

At the end of the procedure, a nephrostomy tube of size $12-16 \mathrm{~F}$ is placed through the tract into the collecting system, large enough to maintain an adequate tract to permit blood and clots to drain readily. After 48 hours, a nephrostogram is obtained by injecting contrast through the nephrostomy tube. If there is no leak, the nephrostomy tube is clamped, and if tolerated, the tube is removed and the patient is discharged from the hospital. The drainage site will usually close within 24 hours. Convalescence time varies; most patients returning to average activity levels within a week or so. Vigorous activity should probably take an extra week.

\section{Indications for PCNL}

Extra corporeal shock wave lithotripsy (ESWL) is the treatment of choice for small and medium sized uncomplicated stones. Following over three decades' experience with ESWL, which has allowed better understanding of its usefulness and limitations, the indications of PCNL have been redefined:

\section{Obstructive uropathy}

If an anatomic abnormality is present that will prevent stone fragments from passing spontaneously, ESWL is usually contraindicated. These situations are ideal for PCNL, in as much as the obstructive uropathy can also be corrected after stone removal employing endourological procedures. Uretero-pelvic junction
(UPJ) obstruction may coexist with calculi in the collecting system. Such stones are best removed by percutaneous means because the obstruction can be treated by endopyelotomy, usually at the same time.

\section{Stone size}

Although it is possible to treat large stones with ESWL, the high re-treatment and residual stone rates make such treatment unattractive [7]. PCNL is particularly effective with these as large volumes of stone material can be removed quickly. Hence, PCNL is the treatment of choice for stones $\geq 2.0 \mathrm{~cm}$ including branched (staghorn) stones.

\section{Anatomic abnormalities}

Patients with a larger body habitus make it difficult for ESWL to target the focal point of the stone. PCNL can be employed in any case where the distance from the skin to the stone is less than the length of the nephroscope or sheath. Similarly, patients with kyphosis or kypho-scoliosis of severe degree are better served by PCNL. The results of ESWL are unsatisfactory in very obese patients, where PCNL can be an effective option.

\section{Stone location}

Stones located in the lower pole calyces are less likely to pass after shock wave breakup, particularly if the collecting system is grossly dilated or otherwise abnormal. If complete clearance is desired, PCNL is preferable.

\section{Stone composition}

Hard stones will frequently not fragment into small enough particles by ESWL for spontaneous passage with minimal discomfort. Although they might be equally difficult to remove with power lithotripsy, it

\begin{tabular}{|c|c|c|}
\hline Stone location & $\begin{array}{c}\text { Stone size } \\
(\mathrm{mm})\end{array}$ & Treatment \\
\hline All, except lower pole $10-20 \mathrm{~mm}$ & $\begin{array}{c}>20 \\
10-20 \\
<10\end{array}$ & $\begin{array}{l}\text { 1. PCNL 2. RIRS / SWL } \\
\text { 1. SWL 2. Endourology (PCNL) } \\
\text { 1. SWL/ RIRS 2. PCNL } \\
\text { Factors facourable for SWL: Yes - SWL / Endourology }\end{array}$ \\
\hline Lower pole $10-20 \mathrm{~mm}$ & $10-20$ & No - 1. Endo urology 2.SWL \\
\hline
\end{tabular}

Table 1. European Association of Urology Guidelines 2010.Treatment algorithm for renal calculi. ${ }^{21}$ 
will be possible to remove the pieces via PCNL, irrespective of fragmenting difficulties. Similarly, PCNL should be first line for complete clearance of soft, matrix stones, which are difficult to fragment with ESWL.

\section{Certainty of the final result}

Residual stones are not acceptable for many patients, for example an airline pilot. They also leave an uncertainty as to whether small fragments might pass spontaneously. The impeccable stone-free rate of PCNL makes it ideal in such situations.

\section{Other modality failure}

As mentioned, shock wave lithotripsy may fail or ureteroscopy may fail. Equally, stones may remain after an open surgical procedure. PCNL is useful in these difficult situations. .

\section{Contraindications of PCNL}

The only absolute contraindications for PCNL are uncorrected bleeding disorders and pregnancy (risk of radiation). The other relative contraindications which may be considered are - anesthesia unsuitability, and stone location making access risky, (e.g. pelvic kidney). By and large, PCNL is possible in majority of the patients, including those considered unsuitable for the other modalities like open surgery and ESWL.

\section{Results}

The advent of ESWL has changed the definition of what constitutes a successful result. There is considerable debate over "clinically insignificant residual fragments" ("CIRF"), referring to broken up fragments of various sizes and their propensity for spontaneous passage. Due to a lack of unanimity as to the precise definition of CIRF, a consensus has emerged now that the only true definition of success is a stone-free state. This point is an important consideration in measuring the effectiveness of PCNL against other methods of stone management.

If results are restricted to the best selected patients, i.e., those with the least difficult stones to access, stone-free rates of 98 to 99 per cent can be achieved. As stone size and complexity of the situation increases, the stone free rate drops to 75 to 80 per cent. Greater effort leads to better results, hence making it a matter of judgment if a residual stone is worth the effort required to remove it.

\section{Complications}

As with any other surgical procedure, problems may complicate any aspect of the percutaneous stone removal.

\section{Complications related to access and tract} dilatation: The ultimate success of the procedure is a function of adequate access. Poor tract placement may make safe, expeditious stone removal impossible. Prudence dictates that a sub-optimal access point should be changed prior to dilatation and lithotripsy. In obese patients, the kidney may be mobile enough that the dilators simply push the kidney away. The dilators should usually be placed no further than the stone itself. The reason for this is that more aggressive dilatation could generate a perforation of the collecting system. Rarely, some event may indicate that the procedure should be stopped at this point. After 48 to 72 hours of nephrostomy tube drainage, bleeding should stop and the tract will be well stabilized, permitting uncomplicated stone removal.

2. Injury to kidney: Optimum access traverses the bulk of the thickness of renal parenchyma to enter the collecting system through one of the calyces. Placement of the tract in a line too medial or lateral may tear the parenchyma. The pedicle or other large branch vessels may be injured if the access tract enters the collecting system medial to the calyces, or if the tract goes beyond the collecting system inadvertently. Proper placement of the tract through the calyx and infundibulum and into the renal pelvis minimizes such a risk.

3. Bleeding: Although a certain amount of bleeding occurs during the procedure, significant blood loss may complicate the situation. Most commonly bleeding is venous. This can be managed by clamping the nephrostomy tube for 30 to 45 minutes, which allows a clot to form in the collecting system, tamponading the bleeding.

Arterial bleeding is a more serious problem. This may occur acutely during lithotripsy (primary) but also may occur in the postoperative period up to a week or 10 
days (secondary). The rapidity of blood loss, its red arterial character, and its lack of response to tamponade suggests injury to a significant vessel. Arteriography should be performed immediately, which will confirm the diagnosis, usually revealing a pseudo-aneurysm, and will also permit treatment by selective embolization of the offending artery.

4. Extravasation: Normal saline should be used as the irrigation fluid to minimize adverse effects when extravasation occurs. When an Amplatz sheath is employed, most of the irrigation fluid flows out the sheath, rendering extravasation less likely. Intravascular extravasation may be suggested by venous bleeding and confirmed by injection of contrast medium. This finding warrants termination of the procedure for the day, preventing absorption of irrigation fluid. Intraperitoneal extravasation is rare, and will happen only if there is peritoneal laceration subsequent to a pelvic tear.

5.Injury to adjuscent structures: The retroperitoneal position of the kidney permits access through a percutaneous window that enables entry into the kidney without trauma to adjacent peritoneal structures. Pathologic states and variations in normal anatomy may however, result in damage to adjacent organs.

A. Pleura: Most of the time, if access is below the 12 th rib and if the kidney is in normal position, it is unlikely that the pleura will be injured. The risk of injury increases if access is required in the upper pole using supracostal puncture. The incidence of pneumothorax has been reported to vary between 1$13 \%$ with supracostal puncture. Minor leaks can heal spontaneously, but a chest tube should be placed if the pneumothorax is significant.

B. Colon: The close proximity of the colon to the kidney, the normal anatomical variations, and the occasional patient with pathologic enlargement of the colon makes this rare event a possibility.

C. Spleen: Very rare, but increased risk if splenomegaly is present.

6. Sepsis: Many patients experience a rise in temperature after stone removal, although true sepsis is uncommon. Preoperative urine culture results will identify the patient who should be treated prior to the procedure using a broad-spectrum antibiotic that covers the common uro-pathogens. Special attention should be paid to those patients with infected stones.

7. Retained fragments: The presence of residual fragments on a post-procedure plain film can be an unwanted finding. Reinsertion of the nephroscope through the tract will permit removal of the stone fragments.

Sometimes stones are extruded through the collecting system or are noted in the perinephric tissues outside the kidney. It is not necessary to remove these stones, as experience has shown them to be clinically unimportant; only causing confusion on subsequent abdominal radiographs.

8. UPJ obstruction: Occasionally follow up studies show partial or complete UPJ obstruction resulting in progressive dilatation of pelvi-caliceal system. This may be either due to pre-existing obstruction missed at the time of operation, or may be the result of trauma to UPJ during surgery. The incidence is greatest in cases where the stone was impacted at the UPJ.

\section{Recent advancements}

As with all aspects of medicine and surgery, we are living in an era of a constantly evolving environment. PCNL surgery as well, is part of this phenomenon. Newer techniques to make this operation more successful in providing better outcomes are continually being strived for. Highlights of such developments are listed.

1. Tubeless PCNL - Conventionally, a nephrostomy tube for post-operative drainage of kidney has been an integral part of the procedure of PCNL [10]. 'Tubeless' PCNL involves placing a double-J stent instead of a nephrostomy tube at the end of the procedure. Many studies have proven its various advantages such as lesser pain and reduced time as an in-patient., Following post-operative imaging to rule out any residual fragments, the double-J stent can be removed by cystoscopy, slight disadvantage over the nephrostomy tube as the patient needs to undergo this additional minor procedure. However, this second can also be eliminated by attaching a tether (e.g. nylon 
string) to the cephalic end of the stent, tunneling it through the nephrostomy tract to the skin. This can then aid in effortless removal of the stent by pulling on the tether, as well as keeping the tract open in place in case a second look is required [11].

2. Flexible nephroscope - a simple yet incredibly effective instrument, the flexible nephroscope provides easy maneuverability within the calyces, allowing good visualization. The flexibility also allows the surgeon to avoid supracostal punctures, and instead make the puncture lower down and then travelling to a higher calyx if needed. The flexibility of the nephroscope helps in accessing calyces not normally visible with a rigid nephroscope, which lets the surgeon check for fragments from all calyces without the need for making multiple tracts [13]. Studies have also shown it to provide higher stonefree rates, lower bleeding, and lower incidences of second look procedures [13].

3. Ultrasound-guided puncture - This aids in the initial and most important step of the procedure the puncture. Combined with traditional fluoroscopy, ultrasound assists the surgeon in determining the precise location, angle and depth of puncture. This minimizes risk of inaccuracy, improving the overall results of the operations, as well as reducing complications such as perforating adjacent structures accidently [14]. Most importantly, it results in significant reduction in radiation exposure to the patient as well as the operating surgeon and his team [14].

4. Supine PCNL - Conventional PCNL involves laying the patient in a prone position for posterior access to the kidney. However, it is coming to light that the same could be achieved by placing the patient in the supine position. This position is especially useful in patients with poor respiratory reserve, including those at the higher end of body mass index, as it aids in easier ventilation of patients. Recent studies have shown the supine approach has lesser complications such as colon perforation, as well as providing a good surface area for access and surgical instrumentation [15]. In addition, the supine approach may also require lesser radiological exposure and reduced operative time. Although this technique sounds like a welcome improvement, it is still rather new, and further studies in the future shall determine its true value [15].

5. Holmium laser - Besides the conventional ultrasonic and pneumatic devices, Holmium laser has also been introduced as an energy source to fragment the stones. Its narrow fiber of $<1 \mathrm{~mm}$ diameter allows use with mini- and micro- instruments, as well as flexible instruments [16]. It also minimizes any accidental firing on the renal mucosa [17]. Although there is no significant reductions in operative time compared to pneumatic lithoclast, it does however have a lower complication rate. Its higher purchasing cost however, limits its use to institutions with the required capital [17].

6. Mini PCNL - Halving the diameter of the nephroscope to $12 \mathrm{~F}$ and using an 15 or $18 \mathrm{~F}$ Amplatz sheath significantly reduces the tissue damage during access [18] This technique improves outcomes, primarily in stones $<2 \mathrm{~cm}$, in aspects such as morbidity and in-patient time. It also eliminates the need of placing a nephrostomy tube, as a double-J stent or indwelling ureteric catheter is sufficient. Although efficacy is higher in smaller stones, further advancements in technique and instrumentation may soon allow successful outcomes in larger stones as well.

7. Micro-perc - The concept of 'micropercutaneous nephrolithotomy', or simply - 'microperc', primarily used for stones $\leq 1 \mathrm{~cm}$, obviates the need for tract dilatation, with the aid of a $4.85 \mathrm{~F}$ "allseeing" needle [20]. It aids in reducing the invasiveness of the surgery, as well as reducing morbidity; whilst still maintaining effective stone clearance. There are some obvious drawbacks associated with this technique, namely reduced visibility due to a narrower sheath, as well as the need to fragment the stones to a smaller size than normal [20]. Its efficacy is also currently limited to stones of the small spectrum; hence the cohort it can benefit is limited at present. 


\section{Summary}

Percutaneous surgery is an effective and safe method for the management of a wide variety of renal and upper ureteral stones. Today, it is used mainly in certain specific situations in which the result justifies its invasive nature. Recognition of which patient can benefit from the procedure minimizes the number of other less successful attempts at stone removal and optimizes the chance that the problem will be managed safely, expeditiously, and economically.

\section{References}

1. Goodwin WE, Casey WC, Woolf W. Percutaneous trocar (needle) nephrostomy in hydronephrosis. J Am Med Assoc 1955;157(11):891-4.

2. Fernstrom I, Johansson B. Percutaneous pyelolithotomy. A new extraction technique. Scand J Urol Nephrol 1976;10(3):257-9.

3. White EC, Smith AD. Percutaneous stone extraction from 200 patients. J Urol 1984;132(3):437-8.

4. Alken P, Hutschenreiter G, Gunther R, Marberger M. Percutaneous stone manipulation. J Urol 1981;125(4):463-6.

5. Wickham JE, Kellett MJ, Miller RA. Elective percutaneous nephrolithotomy in 50 patients: an analysis of the technique, results and complications. J Urol 1983;129(5):904-6.

6. Segura JW, Patterson DE, LeRoy AJ, May GR, Smith LH. Percutaneous lithotripsy. J Urol 1983;130(6):1051-4.

7. Lingeman JE, Smith LH, Woods JR, Newman DM. URINARY CALCULI ESWL ENDOUROLOGY AND MEDICAL THERAPY. Philadelphia, Pennsylvania, USA; London, England, UK: Lea and Febiger, 1989. $\mathrm{Xvi}+460 \mathrm{p}$.

8. Brannen GE, Bush WH, Correa RJ, Gibbons RP, Elder JS. Kidney stone removal: percutaneous versus surgical lithotomy. J Urol 1985;133(1):6-12.

9. Segura JW, Patterson DE, LeRoy AJ, Williams HJ, Jr., Barrett DM, Benson RC, Jr., et al. Percutaneous removal of kidney stones: review of 1,000 cases. J Urol 1985;134(6):1077-81.

10. Agrawal MS, Agrawal M. Are multiple nephrostomy tubes necessary after multitract percutaneous nephrolithotomy? A randomized comparison of single versus multiple nephrostomy tubes. J Endourol 2009;23(11):1831-4.

11. Agrawal MS, Agrawal M. Tubeless percutaneous nephrolithotomy. Indian journal of urology : IJU : journal of the Urological Society of India 2010;26(1):16-24.

12. Agrawal MS, Agrawal M, Gupta A, Bansal S, Yadav A, Goyal J. A randomized comparison of tubeless and standard percutaneous nephrolithotomy. J Endourol 2008;22(3):43942.

13. Gucuk A, Kemahli E, Uyeturk U, Tuygun C, Yildiz M, Metin A. Routine Flexible Nephroscopy for Percutaneous Nephrolithotomy for Renal Stones with Low Density: A Prospective, Randomized Study. Journal of Urology 2013;190(1):144-8.

14. Agarwal M, Agrawal MS, Jaiswal A, Kumar D, Yadav H, Lavania P. Safety and efficacy of ultrasonography as an adjunct to fluoroscopy for renal access in percutaneous nephrolithotomy (PCNL). BJU international 2011;108(8):1346-9.

15. Vicentini FC, Torricelli FCM, Mazzucchi E, Hisano M, Murta CB, Danilovic A, et al. Modified complete supine percutaneous nephrolithotomy: solving some problems. Journal of endourology / Endourological Society 2013;27(7):845-9.

16. Zhang X, Yu JJ, Yang RX. Minimally Invasive Management with Holmium Laser in Total Urinary Tract Calculi. Photomedicine and Laser Surgery 2013;31(5):230-5.

17. Malik HA, Tipu SA, Mohayuddin N, Sultan G, Hussain M, Hashmi A, et al. Comparison of holmium: Yag laser and pneumatic lithoclast in percutaneous nephrolithotomy. JPMA The Journal of the Pakistan Medical Association 2007;57(8):385-7.

18. Abdelhafez MF, Amend B, Bedke J, Kruck S, Nagele U, Stenzl A, et al. Minimally Invasive Percutaneous Nephrolithotomy: A Comparative Study of the Management of Small and Large Renal Stones. Urology 2013;81(2):241-5.

19. Mishra S, Sharma R, Garg C, Kurien A, Sabnis R, Desai M. Prospective comparative study of miniperc and standard PNL for treatment of 1 to $2 \mathrm{~cm}$ size renal stone. BJU international 2011;108(6):896-9; discussion 9-900. 
20. Armagan A, Tepeler A, Silay MS, Ersoz C, Akcay M, Akman T, et al. Micropercutaneous nephrolithotomy in the treatment of moderatesize renal calculi. Journal of endourology / Endourological Society 2013;27(2):177-81.

21. C. Türk, T. Knoll, A. Petrik, K. Sarica, A. Skolarikos, M. Straub, C. Seitz ; members of the European Association of Urology (EAU) Guidelines Office. Guidelines on Urolithiasis. In: EAU Guidelines, edition presented at the 25th EAU Annual Congress, Barcelona 2010. ISBN 978-90-70754-71-7. 